\title{
Study of RPCs for autonomous field stations in cosmic ray research
}

\author{
Luis Lopes ${ }^{1}$ \\ Laboratório de Instrumentação e Física Experimental de Partículas (LIP) \\ Departamento de Física da Universidade de Coimbra, 3004-516 Coimbra, Portugal \\ E-mail: luisalberto@coimbra.lip.pt
}

\section{Paulo Fonte}

Laboratório de Instrumentação e Física Experimental de Partículas (LIP)

Departamento de Física da Universidade de Coimbra, 3004-516 Coimbra, Portugal

E-mail: fonte@coimbra.lip.pt

\section{Mário Pimenta}

Laboratório de Instrumentação e Física Experimental de Partículas (LIP)

Avenida Elias Garcia 14, 1º, 1000-149 Lisboa, Portugal

E-mail: pimenta@lip.pt

\begin{abstract}
The capability of covering very large areas at low cost, besides showing excellent performance in many aspects, motivated the application of RPCs to Nuclear and High Energy Physics and also to Cosmic Ray research in experiments such as COVER-PLASTEX and ARGO/YBJ. Such detectors, however, require indoor conditions and support systems. For very high energy cosmic ray research, where shower sampling is mandatory, it would be convenient to develop detectors that could be deployed in small standalone stations, with very sparse opportunities for maintenance, and with good resilience to environmental conditions. With this aim we developed glass RPCs that are confined to a sealed plastic box housing all high voltage and gas distribution. The detector is impervious to humidity and requires only $0.4 \mathrm{cc} / \mathrm{min}$ of gas flow rate, equivalent to $1 \mathrm{~kg} /$ year of R-134a. Arbitrary readout electrodes can be applied externally.
\end{abstract}

XI workshop on Resistive Plate Chambers and Related Detectors (RPC2012)

INFN-Laboratori Nazionali di Frascait, Italy

February 5-10, 2012

\section{Introdution}

The abundance of available literature confirms that Resistive Plate Chambers [1] have been applied with great success in many High Energy and Nuclear Physics experiments over the

\footnotetext{
$1 \quad$ Speaker
} 
last two decades. In cosmic ray physics, RPCs have been already used in the COVER-PLASTEX [2] and ARGO/YBJ [3] experiments.

The previous referred experiments are all running indoors, where all environment variables are continuously monitored and/or adjusted assuring that the detectors will operate under safe conditions. When outdoors, in an uncontrolled environment, large daily temperature and humidity variations should be accommodated. High voltage insulation and gas tightness should be assured very reliably. The chambers must be able to operate for long periods of time without maintenance, calling for a much reduced gas flow. The present work tries to address some of these issues. We first assure the correct indoor operation of the prototype and show some preliminary outdoor results.

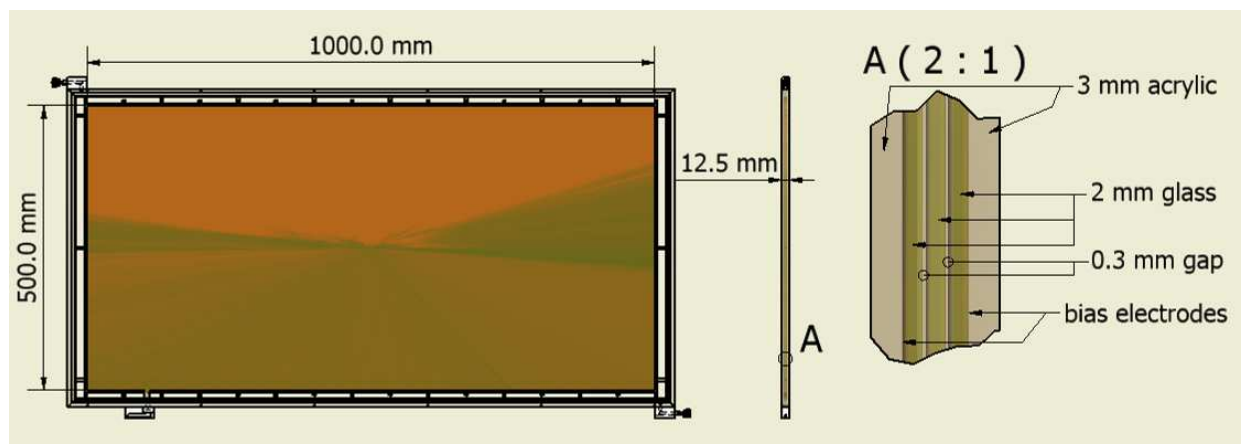

Figure 1: Schematic drawing of the detection module. Three $1000 \times 500 \times 2 \mathrm{~mm}^{3}$ glass plates define two $0.3 \mathrm{~mm}$ gas gaps. The high voltage is applied by means of a layer of resistive acrylic paint on the outer glass electrodes. Gas tightness and high voltage insulation is provided by the permanently glued acrylic box.

\section{Prototype development}

The design of the detector follows an approach in which the sensitive volume is physically separated from the signal pickup electrodes [4-6]. The main idea behind this approach is to solve at once the high voltage insulation and gas tightness issues. This will considerably reduce the amount of feedthroughs, thus easing the achievement of a gas tight volume and also decouples the high voltage from the front end electronics.

The detection module (fig.1) consists in two $0.3 \mathrm{~mm}$ gas gaps defined by $1000 \times 500 \times 2 \mathrm{~mm}^{3}$ glass electrodes separated by Mylar monofilaments (phishing line). The stack is then closed inside a permanently glued acrylic box. The high voltage is applied by means of a layer of resistive acrylic paint [7] on the outer glass electrodes. Only four feedthroughs are needed, two for the high voltage and two for gas input and output.

Since for remote outdoor applications complex gas systems are undesirable, all tests were performed using pure R-134a (tetrafluoretane). In a first moment it is used at a flow rate of $10 \mathrm{cc} / \mathrm{min}$ to renew the gas volume and after stabilization the flow rate is reduced to $0.4 \mathrm{cc} / \mathrm{min}$. Previous measurements [8] confirm the possibility to operate RPCs with pure tetrafluoretane 
with only a small decrease in the efficiency when compared with binary and ternary mixtures. This could be compensated by increasing the number of gaps or the gap width, depending on the timing requirements.
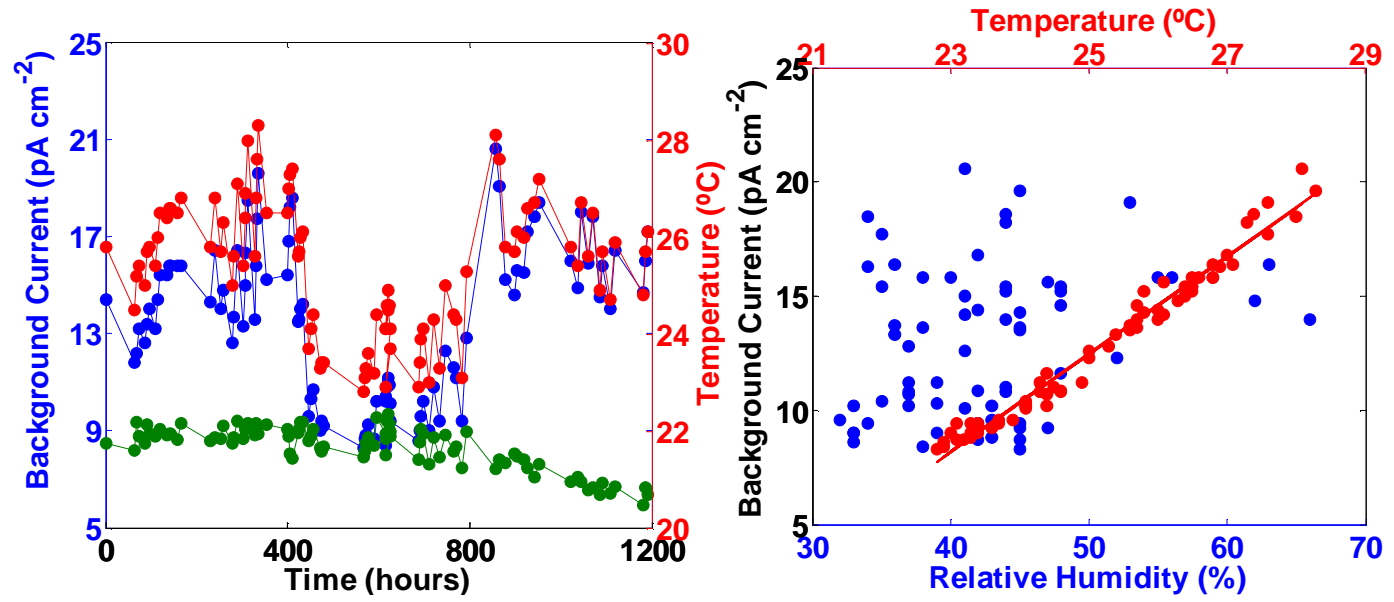

Figure 2: Left panel: background current at a constant gas flow rate of $0.4 \mathrm{cc} / \mathrm{min}$ (blue line), showing also the temperature (red line) and the current normalized to a temperature of $23{ }^{\circ} \mathrm{C}$ (green line). Right panel: background current vs. ambient temperature (top x scale, red) and background current vs. relative humidity (bottom x scale, blue). The background current is well correlated with temperature but it is unaffected by relative humidity.

\section{Indoor measurements}

Before testing the chamber outdoors some measurements of the relevant parameters were carried out in the laboratory. We consider the dark current to be the most relevant single parameter to easily monitor the correct operation of this kind of detector. Since the variations in the ambient pressure (few tens of mbar) have very little effect on the detector gas gain, we only consider at this stage the possible influence of temperature and relative humidity.

\subsection{Dark Current}

After a conditioning period (20 days, not shown in the plots) at $10 \mathrm{cc} / \mathrm{min}$ the gas flow rate was reduced to $0.4 \mathrm{cc} / \mathrm{min}$. The working point was set to $5600 \mathrm{~V}$ ( $2800 \mathrm{~V} / \mathrm{gap})$.

In fig. 2 (left panel) it is shown the background current (blue) and the temperature (red) variations along 50 days. It is apparent that the background current is very well correlated with the ambient temperature. This is further illustrated in fig. 2 (right panel) were we plot the background current as a function of the temperature (top $\mathrm{x}$ scale, red). The green curve in the left panel show the background current corrected by the temperature for a reference temperature of $23{ }^{\circ} \mathrm{C}$. The stability of the current at a constant temperature demonstrates the operation of the chamber at a very low gas flow rate, equivalent to $1 \mathrm{~kg} / \mathrm{year}$.

Acrylic has a very small but not negligible water absorption coefficient [9], consequently it is wise to look for the influence of moisture on the performance of the detector. In fig. 2 (right 
panel) we plot the background current as function of relative humidity (bottom x scale, blue), showing no correlation between them. To further this observation we performed a quite radical test (fig. 3) placing the chamber underwater while keeping the HV on for more than 15 days, observing no increase in the background current.

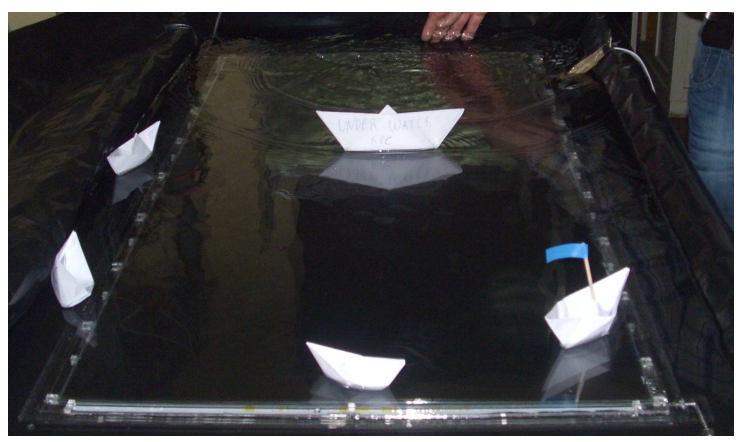

Figure 3: Humidity sensitivity test with the chamber underwater with HV applied for 15 days, confirming that the detector is quite impervious to humidity.

\subsection{Efficiency for cosmic rays, charge spectra and streamer fraction}

The setup to measure the efficiency consists of two external plastic scintillators and a small trigger RPC with $99 \%$ efficiency to cosmic rays. The test area defined by the setup is around $12 \mathrm{~cm}^{2}$. The chamber in test is placed between the trigger RPC and the bottom scintillator. Between both RPCs it was placed a lead block with $5 \mathrm{~cm}$ thickness to absorb the softer muons. Two square metallic electrodes with $100 \mathrm{~cm}^{2}$ were used to collect the signal from the detector to a slow charge amplifier with $10 \mu \mathrm{s}$ CR-RC shaping. The trigger is generated from the coincidence between the two scintillators and the trigger RPC. The signals were recorded by a digital oscilloscope ( $1 \mathrm{GHz} \mathrm{BW}, 400 \mathrm{ps} / \mathrm{sample}$ ) and analyzed by software.

In fig. 4 (left panel) we plot the efficiency as a function of the applied voltage. In black we have the extrapolated curve from an equivalent measurement made with a single gap RPC [8]: the expected efficiency at the plateau should be around $87 \%$ for our double gap RPC. We are able to reach the expected efficiency, however at a slightly higher applied voltage. Higher efficiency values can be achieved by increasing the number of gaps or/and the gap width, depending on the timing requirements.

The observed charge spectra of all efficient events (fig. 4, right panel) show a well defined separation between avalanches and streamers. The avalanche's peak moves to the right as the high voltage increases, showing a clear decrease on the contribution of the smaller events. Also from the observation of the charge spectra the streamers fraction as function of the applied voltage can be evaluated. We define $10 \%$ as the operability limit, which was reached for $6000 \mathrm{~V}$ (3000 V/gap). So we were able to operate the chamber at the efficiency plateau (400 V wide) with less then $10 \%$ of streamers, reinforcing the impressions of chamber good health and robustness. 

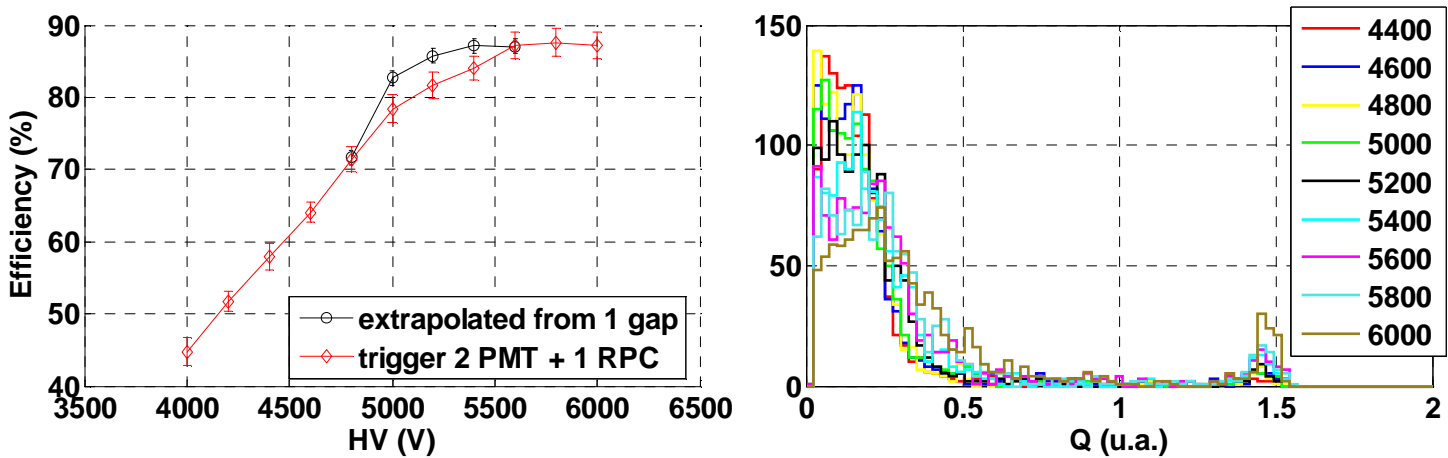

Figure 4: Left panel: efficiency vs. applied voltage. We achieve the expected efficiency of $87 \%$ for a double gap RPC filled with pure R-134a. The black curve is the value extrapolated from single-gap measurements. Right panel: charge spectrum, the minimum is the efficiency threshold; the avalanches are well separated from the streamers. The avalanche's peak moves to the right as the high voltage increases.

\section{Outdoor}

In order to protect the chamber from the more pronounced temperature variations found in outdoor conditions we built a "thermal amplitude reduction box". The aim is to reach inside this box a temperature variation similar to the one found in the laboratory, or, at least, an equivalent daily gradient. This is because from laboratory measurements it is known that the efficiency plateau of $200 \mathrm{~V} / \mathrm{gap}$ absorbs the $25^{\circ} \mathrm{C}$ daily variation in the temperature without affecting the detector performance, at least for the observed cosmic rays counting rates.

In fig. 5 it is shown the background current (top panel) and the temperature inside the box (bottom panel) both as function of time, for a chamber operating at a nominal voltage of $5600 \mathrm{~V}$ and at a gas flow rate of $0.4 \mathrm{cc} / \mathrm{min}$. The chamber as been on for more than four months without registering any abnormal behaviour. The background current did not increase and it seems very well correlated with temperature, as in the laboratory. We also recorded the ambient pressure and relative humidity and no correlation between these and the current was found. More operation time is needed to mature these observations.

\section{Conclusion}

We developed glass RPCs that are confined to a sealed plastic box housing all high voltage and gas distribution. The detector is impervious to humidity and requires only $0.4 \mathrm{cc} / \mathrm{min}$ of gas, equivalent to $1 \mathrm{~kg} / \mathrm{year}$ of R-134a. Arbitrary readout electrodes can be applied externally.

The observations made indoor and outdoor suggest a well-performing detector. We intend to verify the range of the efficiency plateau as function of temperature and perform also measurements of the time resolution.

Outdoor tests will continue as well. The next steps are the monitoring of the practical quantities, such as efficiency, time resolution and streamers fraction, aiming to find any correlation between these and the environmental conditions. 


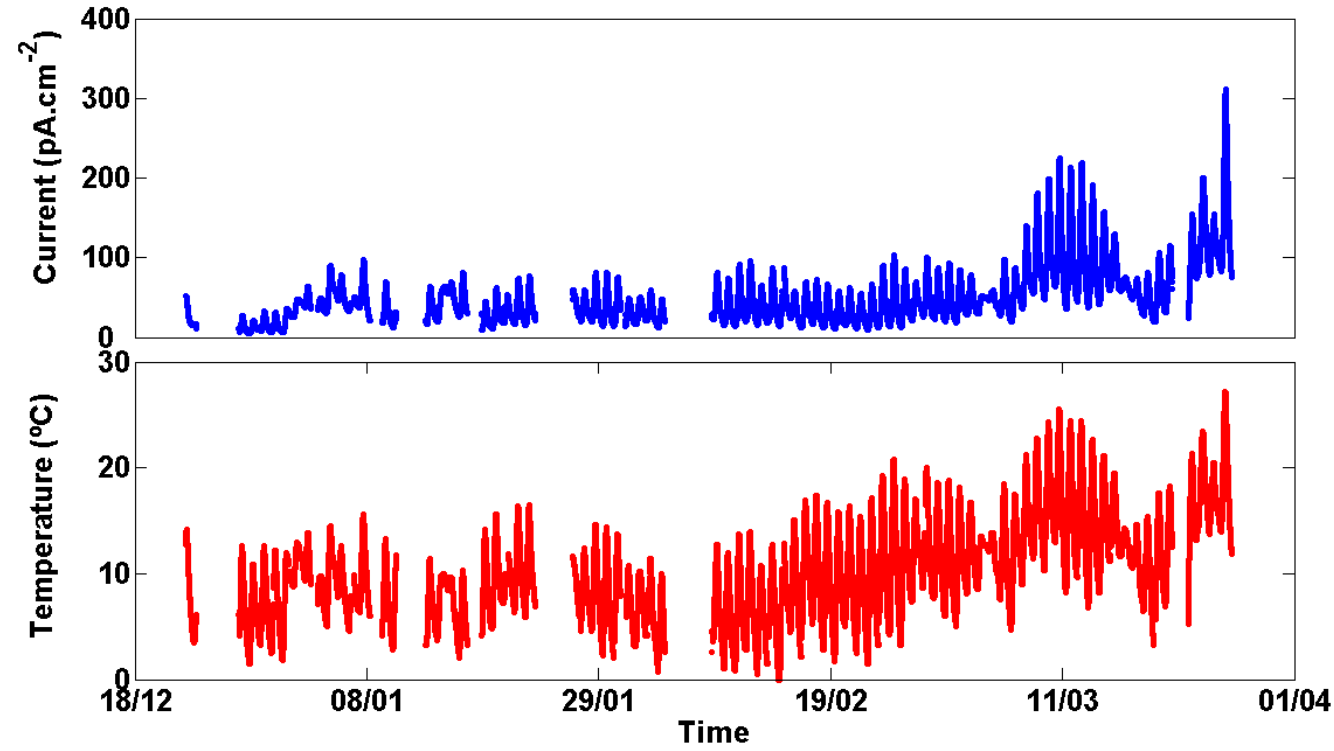

Figure 5: Top panel: background current vs. time, outdoors, with reduced gas flow: no systematic increase is observed along the operation time. Bottom panel: temperature inside the box vs. time, showing that the background current follows the temperature variation.

\section{Acknowledgements}

Portuguese national funds and FEDER funds within COMPETE - Programa Operacional Factores de Competitividade through Fundação para a Ciência e a Tecnologia, Portugal

\section{References}

[1] R.Santonico and R. Cardarelli, Nucl. Instr. and Meth., 1981, 187:377.

[2] G.A. Agnetta et al., Nucl. Instr. and Meth. A, 1996, 381: 64-72.

[3] G. Aielli et al., Nucl. Instr. and Meth. A, 2006 562: 92-96.

[4] G. Cattani et al., Nuclear Instr. and Meth. in Phys. Res. A661 (2012), S6-S9.

[5] S. Park et al., Nuclear Instr. and Meth. in Phys. Res. A661 (2012), S82-S85.

[6] R. Antolini et al., Radiation Measurements 44 (2009), 926-928.

[7] L. Xia et al., International Journal of Modern Physics A20, No. 16 (2005), 3830-3833.

[8] L. Lopes et al., Nuclear Instr. and Meth. in Phys. Res. A661 (2012), S194-S197.

[9] http://www.boedeker.com/acryl_p.htm 